\title{
Cryo-section Dissection of the Adult Subependymal Zone for Accurate and Deep Quantitative Proteome Analysis
}

\author{
Christian Friess ${ }^{1}$, Magdalena Götz ${ }^{1,2,3}$, Jacob Kjell ${ }^{1,2,4}$ \\ ${ }^{1}$ Division of Physiological Genomics, Biomedical Center, Ludwig Maximilian University of Munich ${ }^{2}$ Institute for Stem Cell Research, Helmholtz Zentrum \\ Munchen ${ }^{3}$ SYNERGY, Excellence Cluster Systems Neurology, University of Munich ${ }^{4}$ Department of Clinical Neuroscience, Karolinska Institutet
}

\section{Corresponding Author}

Jacob Kjell

jacob.kjell@ki.se

\section{Citation}

Friess, C., Götz, M., Kjell, J. Cryosection Dissection of the Adult Subependymal Zone for Accurate and Deep Quantitative Proteome Analysis. J. Vis. Exp. (176), e63047, doi:10.3791/63047 (2021).

\section{Date Published}

October 7, 2021

\section{DOI}

$10.3791 / 63047$

\section{URL}

jove.com/video/63047

\section{Abstract}

The subependymal neurogenic niche consists of a paraventricular ribbon of the lateral ventricular wall of the lateral ventricle. The subependymal zone (SEZ) is a thin and distinct region exposed to the ventricles and cerebrospinal fluid. The isolation of this niche allows the analysis of a neurogenic stem cell microenvironment. However, extraction of small tissues for proteome analysis is challenging, especially for the maintenance of considerable measurement depth and the achievement of reliable robustness. A new method termed cryo-section-dissection (CSD), combining high precision with minimal tissue perturbation, was developed to address these challenges. The method is compatible with state-of-the-art mass spectrometry (MS) methods that allow the detection of low-abundant niche regulators. This study compared the CSD and its proteome data to the method and data obtained by lasercapture-microdissection (LCM) and a standard wholemount dissection. The CSD method resulted in twice the quantification depth in less than half the preparation time compared to the LCM and simultaneously clearly outperformed the dissection precision of the wholemount dissection. Hence, CSD is a superior method for collecting the SEZ for proteome analysis.

\section{Introduction}

As neurogenesis is restricted in the adult brain, various central nervous system repair strategies would greatly benefit from an increased understanding of the underpinnings of adult neural replacement. Rodents have helped us understand the basic mechanisms of postnatal neurogenesis, although it should be noted that adult neurogenesis is greatly speciesdependent. In mice, there are three adult neural stem cell
(NSC) niches. The hypothalamus is an adult NSC niche with neurogenic potential ${ }^{1,2}$, while continuous adult neurogenesis is mainly restricted to the hippocampus ${ }^{3}$ and the SEZ of the lateral walls of the lateral ventricles ${ }^{4,5,6}$. The SEZ is the largest germinal region containing NSCs (type B cells) that develop into neuroblasts (type A cells) via transit-amplifying progenitor cells (type C cells). The SEZ contains $20-35 \%$ 
of type B cells, $1-15 \%$ of type C cells, $1-30 \%$ of type A cells, and $25-50 \%$ of ependymal cells ${ }^{7}$. The SEZ features a complex microarchitecture, with endothelial cells, microglial cells, and ependymal cells residing in and influencing the stem cell niche $8,9,10$. Although neurons are scarce in the SEZ, axons emanating from distant sources such as the striatum, the ventral tegmental area, or the hypothalamus reach and influence type $B$ cells ${ }^{4}$. A unique feature of this stem cell niche is the separation between the site of proliferation and the site of differentiation. After proliferation, the neuronal progenitors migrate several millimeters from the SEZ to the olfactory bulb, where they terminally differentiate into neurons and integrate into pre-existing neural circuits. Investigations into cell-intrinsic programs associated with neurogenesis have already provided knowledge important for experimental therapeutic cell reprogramming and transplantation strategies $15,16,17,18,19,20$. However, cellextrinsic signals also regulate neurogenesis, and tissue environments can determine the neurogenic fate of stem cells ${ }^{11,12,14,21,22,23}$. Consequently, the investigation of the microenvironment of the neurogenic niches and its interaction with the stem cells is of crucial importance.

The extracellular matrix (ECM) and other secreted proteins are a large part of the microenvironment. For accurate identification and quantification, a proteomic approach is better suited than a transcriptomic approach to determine ECM composition due to the low correlation between transcriptome and protein levels for $\mathrm{ECM}^{24,25}$. Moreover, there is substantial evidence that niche regulators in the SEZ are not exclusively produced by cells populating the niche itself. More distant locations, such as the choroid plexus, secrete modulatory signals transmitted to the stem cells via the cerebrospinal fluid 22,23 . Investigating the niche proteome can help to identify niche regulators present in the niche independent of their production site, given that a substantial proportion of the extracellular microenvironment is assembled by proteins.

To collect the murine ventricular zone for unbiased proteomic analysis, a method with high precision is required, capturing the ca. $50 \mu \mathrm{m}$ thin paraventricular ribbon containing stem cells while excluding the tissue of the adjacent striatum. Furthermore, tissue perturbation during the dissection must be minimalized for analyzing the extracellular microenvironment because soluble proteins, including growth factors or cytokines, could be washed away easily. Although it is possible to analyze the mass spectra of fixed tissue, the required agent, such as paraformaldehyde, will reduce the protein identification depth and may introduce posttranslational modifications. A common wholemount SEZ dissection, e.g., for the collection of cells for fluorescenceactivated cell sorting analysis, removes the whole SEZ with scissors $^{26}$. This standard dissection is fast with minimal tissue perturbation. However, striatal contamination of the samples cannot be avoided. Conversely, LCM has the outstanding advantage of superior dissection precision. However, LCM may introduce tissue perturbations, for instance, due to background staining or laser-caused protein denaturation. To combine the strengths of the wholemount dissection and LCM, a novel method that is compatible with MS, termed cryo-section-dissection (CSD), was developed (Figure 1A-D). The CSD allows the extraction of the SEZ and the dissection of the SEZ of the medial walls of the lateral ventricles (MEZ), which is an ideal, mostly nonneurogenic control region for the SEZ (see the protocol). The niche proteome obtained by the combination of CSD and state-of-the-art MS methods proved to be useful for the characterization and identification of novel regulators in this 
adult NSC niche ${ }^{25}$. Hence, this method will be useful for the determination of SEZ tissue protein composition.

\section{Protocol}

All experimental procedures in this study were performed in accordance with German and European Union guidelines and were approved by the institutional animal care committee and the government of upper Bavaria (Regierung von Oberbayern). Only male C57BI6 mice between the ages of 8-10 weeks were used for the experiments.

\section{Preparation of the mouse brain ( $15 \mathrm{~min}$ per mouse)}

1. Prepare the dissection medium by adding $5 \mathrm{~mL}$ of $1 \mathrm{M}$ HEPES (final concentration $10 \mathrm{mM}$ ) to $500 \mathrm{~mL}$ of $1 \mathrm{x}$ Hank's Balanced Salt Solution (HBSS).

NOTE: The storage time of the dissection medium (+4 ${ }^{\circ} \mathrm{C}$ ) should not exceed 2 weeks.

2. Sacrifice the mice by cervical dislocation and carefully dissect the brain.

NOTE: When investigating the ECM, the tissue should preferably be unmodified. Cervical dislocation keeps dissection time as short as possible, thereby preventing post-mortal enzymatic autodigestion as much as possible. If removal of blood is critical to the research question, simply perfuse the mouse transcardially with phosphate-buffered saline (PBS) before removing the brain.

3. Extract the brain by manual dissection and place it in a culture dish containing ice-cold dissection medium (Figure 1B - 1).

NOTE: Keep the brains in dissection medium on ice throughout the dissection.
4. Remove the olfactory bulb (OB) with a scalpel (Figure 1B - 2) by a straight coronal cut between the $O B$ and the anterior pole of the cortex.

5. Remove the anterior pole of the cortex with the scalpel using a coronal cut to make the lateral ventricles visible in the coronal plane (Figure 1B - 3).

NOTE: Make sure the coronal cut is made $\sim 5 \mathrm{~mm}$ rostrally from the optic chiasm; otherwise, the rostral part of the SEZ/MEZ will be lost.

6. Using scissors, open both lateral ventricles from the top, starting with a sagittal section from the cortical surface to the ventricular lumen, and elongate this cut in a c-shaped manner following the ventricular flexion (Figure 1B - 4).

7. Connect the caudal ends of the left and right sagittal incision by means of an additional coronal cut with the scissors.

NOTE: The three cuts now form a trapezoid and will facilitate the removal of the cortex and corpus callosum in the next step.

8. Remove the cortex and corpus callosum covering the lateral ventricles using forceps (Figure 1B - 5). Then, remove the cortex and corpus callosum that cover the medial ventricular walls. Here, make additional cuts if the tissue is attached to the medial ventricular walls, or simply lift the cortex and corpus callosum with scissors to dislodge the tissue.

9. Carefully spread the ventricular walls with forceps (Figure 1B - 6). Remove the choroid plexus with forceps. NOTE: Complete removal of the choroid plexus is important to avoid interference with the following dissection steps and avoid potential contamination of the SEZ/MEZ samples. 
10. Put the brain on a glass slide and place the glass slide on top of dry ice to freeze the brain. Maintain the ventricular walls in the open configuration.

NOTE: Ensure enough distance between the lateral and medial walls of the ventricle to facilitate precise and exclusive dissection of SEZ and MEZ. If the tissue contracts back into a closed configuration, use the forceps to fix the walls in the desired position during freezing. Avoid any damage to the SEZ/MEZ. Try applying minimal force, mainly at the top edge of the opened ventricles.

\section{Sectioning of the prepared brain ( $15 \mathrm{~min}$ per mouse)}

1. Cut $50-100 \mu \mathrm{m}$ thick coronal sections of the brain until the end of the lateral ventricle using a cryostat and mount the sections onto glass slides. Ensure that the brain is attached to the cryostat attachment plate at the hindbrain with OCT medium and that no OCT comes in contact with the forebrain, especially at the ventricles.

NOTE: OCT medium will interfere with MS measurements. However, if the tissue will be used for an antibody assay, it is unnecessary to exclude OCT medium. The use of coated glass slides is not recommended. Coated slides apply too much adhesive force on the tissue, thereby impeding the translocation of the tissue specimen from the slides into the microcentrifuge tube in the following steps.

\section{Free-hand dissection of brain slices $(\sim 30 \mathrm{~min}$ per mouse)}

1. Place the glass slides with the brain sections on dry ice under a dissection microscope (Figure 1C - 1).
2. Prepare the microcentrifuge tubes on dry ice, and ensure that the tubes stay on dry ice for at least $1 \mathrm{~min}$ to be sufficiently cold before specimen transfer.

NOTE: Use microcentrifuge tubes of high quality, as some low-quality tubes may shed plastic in the subsequent tissue digestion steps associated with MS measurements.

3. Lift the slices from the dry ice for $15-30 \mathrm{~s}$ to achieve a brief, incomplete thawing to render the compact myelin of the striatum observable as dense white dots.

NOTE: Locating the border between the SEZ and the striatum becomes feasible (Figure 1C - 2, see Figure 2A for the exclusion of myelin and a comparison with the wholemount method). If thawing takes too long, the process can be accelerated by pressing a glove-covered finger onto the opposite side of the glass slide. However, this maneuver should be practiced as excessive thawing occurs easily.

4. Separate the SEZ with a precooled scalpel from the adjacent striatum (Figure 1C,D).

5. Transfer the SEZ either as a whole piece or sectioned into 2-4 parts into a microcentrifuge tube by using the blunt edge of the cooled scalpel. If the tissue is to be used for another type of analysis other than MS, transfer the tissue specimen into the appropriate container instead (e.g., a 96-well plate).

NOTE: Cutting the completely frozen tissue may lead to tissue rapidly breaking away and falling off the slide. Cutting completely thawed tissue leads to the disintegration of the tissue. Ensure that the tissue is neither completely frozen nor completely thawed. 


\section{Representative Results}

When following the above steps, the tissue samples in the microcentrifuge tubes are ready for and compatible with MS sample preparation. After sample preparation, we obtained 5-7 $\mu \mathrm{g}$ of peptides per sample of either SEZ or MEZ per mouse. However, the final amounts of the peptides may depend on the MS preparation method. In the proteome comparisons below, protein identification and quantification depth (500-1,000 proteins per sample) were increased by computationally matching the peptide spectra to peptide spectra libraries created for each tissue region ${ }^{25,27}$. Notably, the loss-less nano fractionation method used here for the creation of the peptide spectra libraries is currently not commercially available. The raw MS data were analyzed using the MaxQuant software ${ }^{28}$, achieving mass accuracies in the parts per billion range ${ }^{29}$. The Max Quant environment allows matching between MS runs. Protein abundance was quantified using a label-free quantification algorithm ${ }^{30}$. Immunohistochemical staining was done on fresh frozen tissues and performed as previously reported ${ }^{25}$ (see the Table of Materials).

\section{Cryo-section-dissection}

The complete SEZ and MEZ of adult mice $(n=4)$ were obtained using CSD (see Figure 1 and protocol). The somatosensory cortex (Cx) was dissected with surgical scissors. Additional 4 mice were dissected in the same manner; however, the dissected tissue was pooled into one sample per region to create the proteome library $(10,923$ identified proteins) for increased protein identification and quantification in the individual samples ${ }^{25}$. In the four individual samples, (mean \pm SD) 6,673 \pm 317.4 proteins were quantified in the SEZ and 6,747 \pm 37.7 in the MEZ. All the MS proteomics data were deposited in the
ProteomeXchange Consortium via the PRIDE $^{31}$ partner repository, and the accession number for the proteomes reported here is ProteomeXchange: PXD016632 (http:// proteomecentral.proteomexchange.org).

\section{Comparison to wholemount dissection}

Wholemount dissection was performed according to a standard protocol $^{26}$. Wholemount dissection revealed a similar number of proteins (approximately 6,000 for SEZ and 6,000 for $C x, n=4$ per group) compared to $C_{S D}{ }^{25}$. One of the intended improvements of using CSD for the SEZ, instead of a wholemount dissection protocol, is the reduction of potential striatal contamination. In SEZ samples contaminated with tissue from another region, detected candidate proteins cannot be allocated to a region as significant enrichment can result from the region of interest and the contaminator. Immunohistochemically, the myelin-associated glycoprotein (MAG) positive myelin-rich internal capsules of the striatum were identified in the wholemount samples but seldom in the CSD samples (Figure 2A). The striatal contamination in the wholemount samples could be confirmed by identifying the enrichment of myelin proteins in the SEZ compared to the somatosensory cortex (Cx) Grey Matter (GM) samples (Figure 2B). Note that large parts of the Cx GM, especially the upper Cx layers, are unmyelinated ${ }^{32}$.

As large fiber bundles pass through the striatum, contamination by this region resulted in the enrichment of myelin proteins compared to the $\mathrm{Cx}$. The myelin proteins used as markers for striatal contamination in the SEZ samples were the myelin basic protein (MBP), the myelinassociated glycoprotein (MAG), the proteolipid-protein 1 (Plp1), and the 2',3'-cyclic-nucleotide 3'-phosphodiesterase (Cnp). All myelin-marker proteins were significantly enriched in the SEZ compared to the Cx. Conversely, comparisons 
for the four myelin marker proteins in the CSD dataset yielded no significant differences when comparing SEZ to Cx (Figure 2B). Proteomic data of the striatum ${ }^{33}$ supports the hypothesis that the enrichment of myelin proteins in the SEZ samples of the wholemount dissection was caused by the contamination with striatal tissue. Hence, the CSD largely prevented contamination by striatal tissue (rich in compact myelin) compared to a wholemount dissection.

Unbiased proteome analysis of non-dissociated tissue can reveal interesting extracellular proteins. With improved dissection using the CSD, extracellular-associated proteins were significantly enriched in the samples compared to the wholemount samples (Figure 2C, annotation enrichment test). The CSD and wholemount dissection display a comparable enrichment of the gene ontology (GO) terms "extracellular vesicular exosome" and "extracellular region part." However, the GO term "Matrisome-associated" is slightly more enriched in the CSD than in the wholemount dissection. Accordingly, the ECM cross-binding enzyme and recently discovered neurogenesis regulator transglutaminase-2 (Tgm2) were found enriched in the SEZ compared to $\mathrm{Cx}$ using the $\mathrm{CSD}^{25}$. In contrast, no difference was found between SEZ and Cx samples obtained by the wholemount dissection (Figure 2D). Proteomic data of the striatum $^{33}$ support the hypothesis that the detection of the neurogenesis regulator Tgm2 by wholemount dissection was impeded by the contamination with striatal tissue. Hence, overall, the cryo-section-dissection is a successful but also necessary improvement to the standard dissection for nichespecific proteome analysis.

\section{Comparison to Laser-capture-microscopy}

The front half of the SEZ and the MEZ of 3 adult mice were obtained for LCM (Figure 3A ). Overall, the LCM method exhibits some disadvantages, specifically regarding tissue perturbation and efficiency. To visualize the region of interest under the dissection microscope, background staining is necessary, potentially washing away small or soluble proteins of interest, e.g., growth factors, cytokines, or ECM regulators such as enzymes. Furthermore, slides spend varying times at room temperature during laser removal. Moreover, the laser itself might denature proteins of interest.

CSD has a considerable advantage over LCM regarding the time and effort necessary to perform the dissection: step 1 of the protocol must be performed similarly for both CSD and LCM; without this step, ventricular walls remain adherent, making the separation of MEZ and SEZ samples difficult. Given that the CSD sections $(100 \mu \mathrm{m})$ are $6-7$ times thicker than the maximum thickness ${ }^{34}$ of the LCM sections $(15 \mu \mathrm{m})$, step 2 (sectioning of the brain) and step 3 (removing the MEZ and SEZ from each coronal section) will take at least 6-7 times longer for LCM. The necessary background staining and setting up the laser microscope will consume additional time. Here, it took three times longer to harvest $50 \%$ of the SEZ and MEZ of 3 animals by LCM compared to $100 \%$ of the SEZ and MEZ of 4 animals by CSD, constituting an eightfold speed advantage of CSD. In summary, LCM not only requires a notable amount of additional effort, but the tissue is also subjected to a substantially longer period of manipulation and temperature changes that can compromise the dynamics and reliability of data generated by subsequent analysis.

The MS results of CSD were compared to the results from the laser capture microdissection (LCM). Both datasets were matched to the proteomic library generated by pooling CSD samples. On average, LCM yielded 3,441 \pm 270.0 and $3,613 \pm 238.7$ individual proteins in the SEZ and medial ventricular zone, respectively (Figure 3B). Given 
the remarkable difference in protein identification, principal component analysis (PCA) displayed distinct separation according to the dissection method (component 1: 62.7\%, not shown). Component 2 displayed the greatest separation for SEZ and MEZ among the LCM samples (8.5\%, Figure 3C). Component 3 also seems to separate LCM and CSD; however, this difference might result from methodbased differences rather than the number of identified proteins $(6.4 \%)$. Nevertheless, the overall regional separation remained strikingly distinct for the cryo-dissection data and vastly better than for LCM. This discrepancy in data dynamics may result from different times spent by the specimens at room temperature during the laser dissection or a higher susceptibility of small tissue amounts to variability in the subsequent proteomics protocols and mass spectrometry measurements.

To search for differences in the proteome profile of the ECM, a 2D annotation enrichment test between CSD and LCM was performed for the SEZ and MEZ (Figure 3D). Calculating the relative enrichment of GO terms between LCM and CSD samples allows the comparison of relative proteome dynamics of the ECM protein clusters between the two methods despite the unequal amount of tissue and the differences in the dissection protocol. The plots reveal a good correlation between LCM and CSD. The annotations "extracellular region part" and "extracellular membrane-bound organelle" are similarly enriched in both methods and regions. Hence, the increased time demand of LCM does not appear to be compensated by a relatively higher sensitivity for ECM-associated proteins. Instead, CSD provides more robust identification/quantification when comparing the sample data for the neurogenesis and SEZ-associated ECM proteins Tgm2, Thrombospondin-4 (Thbs4), S100a6, and Tenacin-C (Tnc) (Figure 3E). In the case of $\mathrm{TnC}$, although quantified in all samples, only CSD displayed enrichment for SEZ compared to MEZ. Nevertheless, the SEZ-associated basal membrane proteins Nidogen-1 (Nid1), Laminin subunit beta-2 (Lamb2), and basement membrane-specific heparan sulfate proteoglycan core protein $(\mathrm{Hspg} 2)^{35}$ displayed an even more robust enrichment in the SEZ (compared to MEZ) in the LCM samples than in the CSD samples (not shown). Hence, CSD can provide tissue samples that provide an accurate and deep quantitative proteome for SEZ characterization in a reasonable timeframe, without worrying about compromised tissue integrity or protein loss.

\section{Statistics}

Statistical testing, 2D annotation enrichment tests, and PCA were done in the Perseus environment. Proteins were included in the analysis if a valid value was detected for each method in at least one sample. Protein abundance and number comparisons were visualized using data analysis software (see the Table of Materials). A permutation-based control of the false discovery rate (FDR) (FDR was set to 0.05 , 250 randomizations) was employed for protein comparisons. For the 2D-annotation enrichment tests ${ }^{36}$, the displayed GO terms are significantly enriched (FDR was set to 0.02 using the Benjamini-Hochberg FDR-control method). 


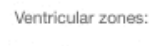

SEZ - Neurogenic

MEZ - Non-neurogenic
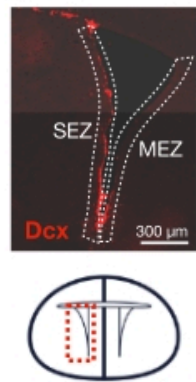

B

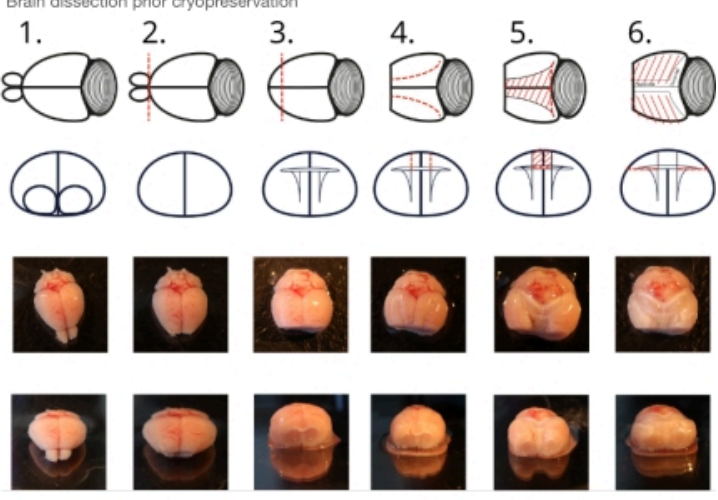

D

SEZ/MEZ dissection confirmation
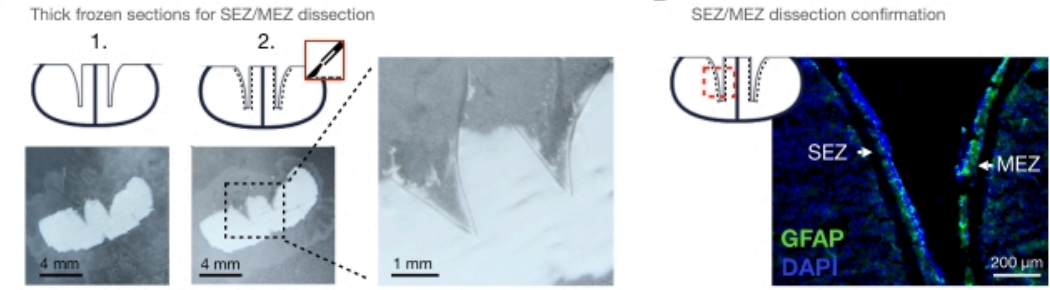

Figure 1: The Cryo-Section-Dissection method. (A) Overview of the region of interest: the lateral ventricle with the neurogenic SEZ and the non-neurogenic MEZ. Neuroblasts immunostained with Dcx. (B) Stepwise removal of the OB, the anterior pole, the cortex, and corpus callosum above the ventricles and the choroid plexus: 1. placement in dissection medium, 2. removal of $\mathrm{OB}, 3$. removal of the anterior pole of the cortex, 4. sagittal incisions of the ventricular top, 5. removal of the ventricular top, 6 . spreading of the ventricular walls. (C) $100 \mu \mathrm{m}$ coronal slices of the fresh-frozen mouse brain, (1.) before and (2.) after the removal of the ventricular walls with an ice-cold scalpel. Scale bars $=4 \mathrm{~mm}$ (D) Staining of a coronal section of a lateral ventricle (GFAP: green; DAPI: blue), showing the SEZ and MEZ dissected with the CSD. Scale bars $=300 \mu \mathrm{m}$ (A), $200 \mu \mathrm{m}$ (D). Abbreviations: CSD = cryo-section dissection; SEZ = subependymal zone; MEZ = medial ependymal zone; Dcx = Doublecortin; OB = olfactory bulb; GFAP = glial fibrillary acidic protein; DAPI = 4',6-diamidino-2phenylindole. Please click here to view a larger version of this figure. 
A

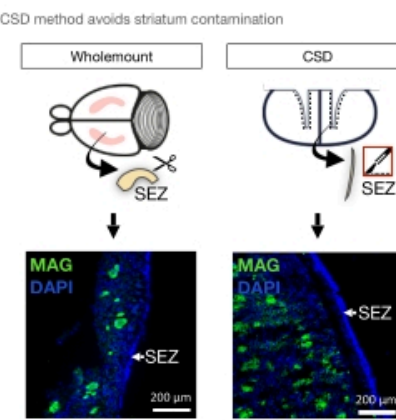

C

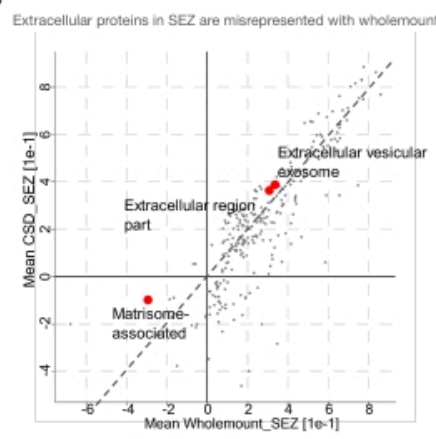

B

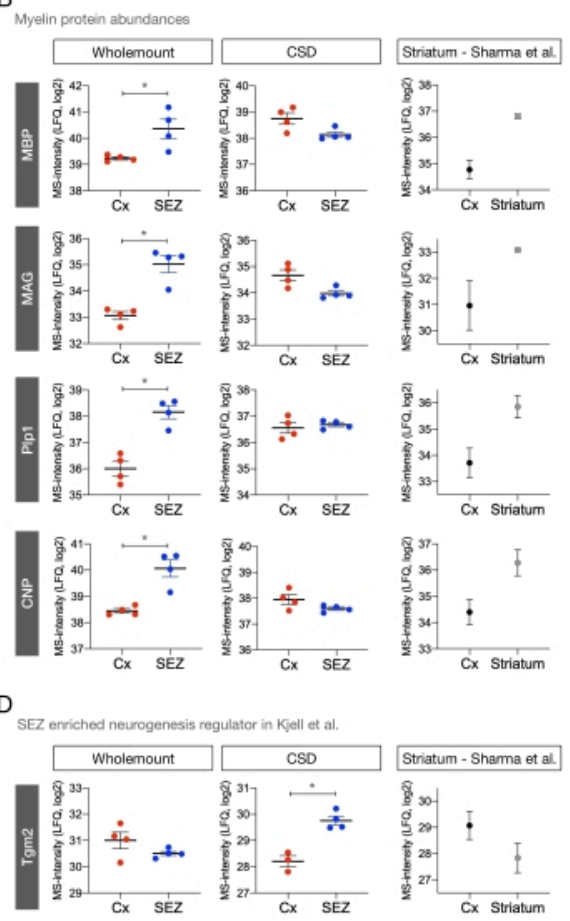

Figure 2: Superior dissection-precision with the cryo-section-dissection compared to wholemount dissection.

(A) Immunohistochemical image of an SEZ sample obtained by wholemount dissection (left). The inclusion of myelin-rich striatal tissue is visualized by staining against MAG (green). Staining of a SEZ dissected with the CSD (right). In CSD, almost all the striatal myelin (staining against MAG, green) is excluded from the sample ribbon. Nuclei were visualized using DAPI (blue). (B) Comparison of myelin marker enrichment in SEZ vs. Cx from wholemount (MBP: $p=0.0074 ;$ MAG: $p=$ 0.0016; Plp1: $p=0.0011 ;$ CNP: $p=0.0029$ ) and CSD (MBP: $p=0.0667 ;$ MAG: $p=0.0236 ;$ Plp1: $p=0.3420 ;$ CNP: $p=$ 0.1842). (C) 2D-annotation enrichment test comparing the wholemount-SEZ with the CSD-SEZ samples. The GO terms extracellular space and Matrisome-associated are more enriched in the CSD data than in the wholemount data. (D) The protein abundance of the NSC regulator Tgm2 ${ }^{25}$ plotted for the wholemount dissection and the CSD. Tgm2 is significantly enriched in the SEZ compared to the Cx in CSD (CSD: $p=0.0029$; Wholemount: $p=0.1775)$. For B and D: As reference, proteome data from Sharma et al. ${ }^{33}$ with measurements of striatum and cortex plotted for the corresponding proteins displayed in the wholemount and CSD samples. Scale bars $=200 \mu \mathrm{m}(\mathrm{A})$. Abbreviations: CSD = cryo-section dissection; SEZ = subependymal zone; $M A G$ = myelin-associated glycoprotein; $\mathrm{Cx}=$ somatosensory cortex; $\mathrm{MBP}=$ myelin basic protein; Plp1 = proteolipid-protein 1; CNP = 2',3'-cyclic-nucleotide 3'-phosphodiesterase; GO = gene ontology; NSC = neural stem cell; Tgm2 = tranglutaminase 2; DAPI = 4',6-diamidino-2-phenylindole; LFQ = label-free quantitation. Please click here to view a larger version of this figure. 
A

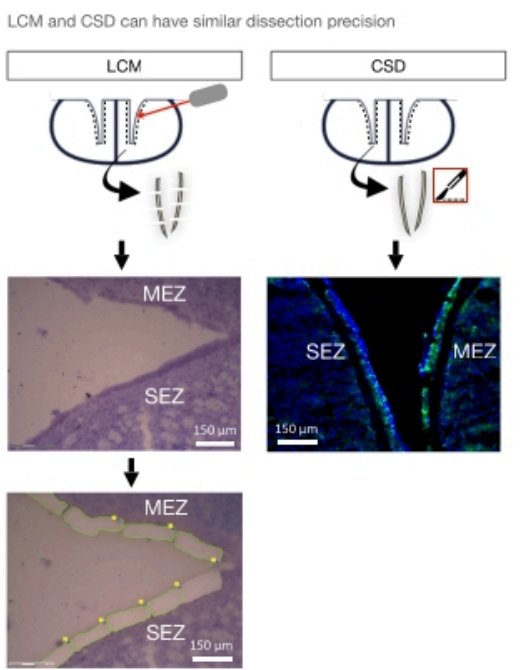

D

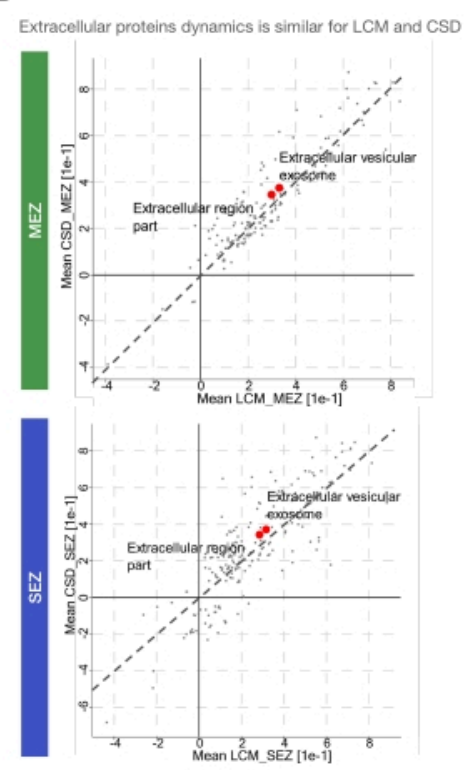

B

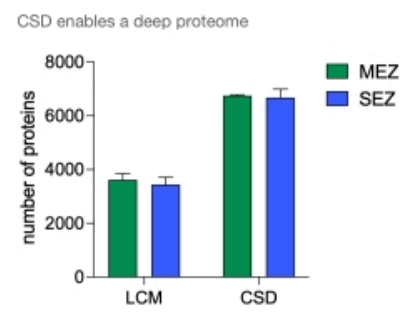

C

Proteome dynamics better define samples in CSD

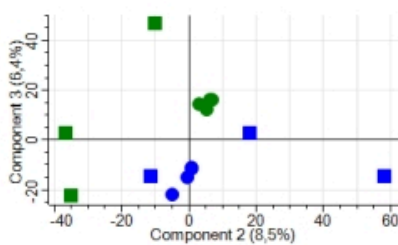

- : CSD $\mathbf{\square}:$ LCM Colors: SEZ MEZ

$\mathrm{E}$
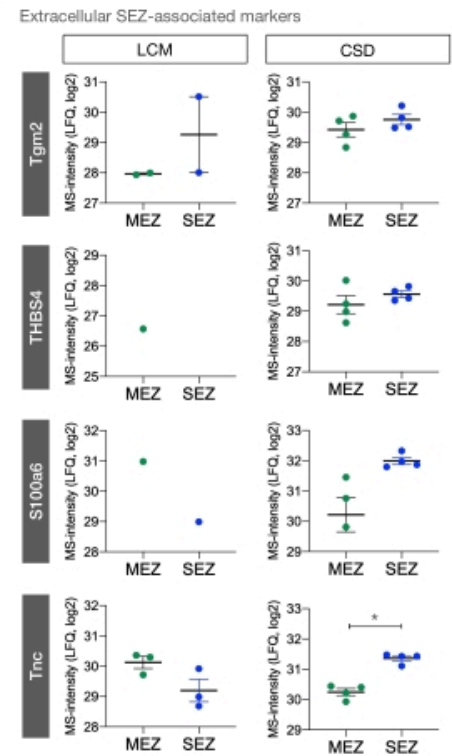

Figure 3: Improved extracellular protein quantification with cryo-section-dissection compared to LCM. (A) Cresyl violet staining of a lateral ventricle before and after laser capture of the SEZ and MEZ (left). For comparison, the CSD incision of the SEZ and MEZ (right). Scale bars $=150 \mu \mathrm{m}$. (B) Comparison of the number of detected proteins in the SEZ and MEZ samples from CSD and LCM. Data are presented as mean \pm SD. (C) Principal component analysis of the SEZ and MEZ samples comparing CSD and LCM (component 2: 8.5\% of the variance; component 3: 6.4\%). (D) 2D annotation enrichment of the cryo-section- and laser-dissected MEZ (Top) and SEZ (Bottom). The GO terms extracellular organelle and extracellular region part are significantly enriched (red dots). (E) Abundances of extracellular SEZ-associated marker proteins in SEZ and MEZ for LCM (Tnc: $p=0.3789$ ) and the CSD samples (Tgm2: $p=0.2940 ;$ S100a6: $p=0.0218$; THBS4: 
$p=0.3941 ;$ Tnc: $p=0.0004)$. Abbreviations: $C S D=$ cryo-section dissection; $L C M=$ laser-capture-microdissection; $S E Z=$ subependymal zone; MEZ = medial ependymal zone; $\mathrm{GO}=$ gene ontology; Tnc = Tenacin-C; Tgm2 = transglutaminase 2; $\mathrm{S} 100 \mathrm{a} 6$ = S100 calcium-binding protein A6; THBS4 = thrombospondin-4; LFQ = label-free quantitation. Please click here to view a larger version of this figure.

\section{Discussion}

The CSD method made it possible to precisely extract SEZ tissue and generate a reliable proteome with significant depth using MS. CSD displays a clear advantage compared to wholemount dissection in terms of greatly reduced striatal contamination of SEZ samples and extracellular protein enrichment. As it is also possible to detect a similar number of proteins in individual samples ( $\sim, 500$ proteins per sample) with CSD and wholemount dissection, the additional time for CSD is well worth the effort. LCM provides more precise SEZ dissection but reached a lower proteome depth, with only 3,500 proteins per sample despite using the same MS protocol as CSD (library matching and labelfree quantification). Importantly, variability was much greater, probably due to the eightfold longer preparation time per sample. PCA of the samples obtained by LCM and CSD reveals a clear separation of both methods with tight regionspecific clusters robustly separated from each other. In contrast, the LCM samples displayed a more scattered distribution, which is probably in part due to the length of preparation. It is unclear whether collecting far more samples over a longer period would have yielded a proteome of equal robustness and depth with LCM. Calculating an estimate, collecting a similar sample volume as done for CSD would take 5-8 times longer with LCM, even up to 15 times longer if samples provided for the peptide spectra libraries were included, and much of it under thawed conditions. Furthermore, considering the additional perturbations of the tissue necessary for LCM (background staining, laser dissection), LCM provided little, if any, gain over CSD. Hence,
CSD can be deemed more suitable for extracellular proteome research, specifically for the SEZ.

Notably, if the region of interest is smaller than the SEZ (e.g., investigating only the ependymal cell layer), a freehand approach falls behind the accuracy of the LCM. For example, using CSD to separate the ependymal from the subependymal layer is difficult as the ependymal layer is only a cell diameter wide, and the demarcation towards the subependymal layer is not visible for the naked eye in fresh frozen tissue. Hence, LCM will be a better choice than CSD if a precise dissection on a scale below $50 \mu \mathrm{m}$ is more important than undisturbed tissue or keeping the dissection time short. For regions with a width of $50 \mu \mathrm{m}$ and more, however, the precision of CSD is comparable to that of LCM for ECM protein analysis.

CSD has already proven to be useful by contributing to the investigation of the functional role of the ECM in the neurogenic niche ${ }^{25}$. Hence, the continued application of CSD in the SEZ for various protein and proteome investigations (or even single-nucleus RNA sequencing) might lead to the detection of further neurogenesis regulators, stem cell activation markers, and a deeper understanding of SEZ stem cell niche physiology. Considering the decline of neurogenesis in the aging SEZ ${ }^{37}$, a concise analysis of ECM changes of the SEZ of aged vs. young mice might promote the understanding of the exact niche mechanisms fostering NSC development and maintenance ${ }^{38,39}$. Furthermore, the influence of inflammation and injury on SEZ neurogenesis is well established $40,41,42,43$. The enrichment of blood- 
derived fibrinogen in the SEZ after cortical brain injury and its influence on SEZ astrogliogenesis and scar formation ${ }^{44}$ highlights the potential influence of traumainduced microenvironment changes on the SEZ stem cell physiology. Hence, investigating the SEZ-ECM proteome in association with brain injury using CSD could help elucidate the mechanisms by which injury and inflammation affect neurogenesis. Importantly, the method could also be applicable to human brain neurogenic niches in health and disease as fresh frozen tissue can often be obtained from surgeries. Furthermore, given the species differences in adult neurogenesis, it would also be fascinating to apply the CSD method to other species, e.g., in association to striatal neurogenesis. Moreover, with other protein detection methods, differences in locally produced growth factors can be investigated accurately and efficiently using CSD for the SEZ and MEZ (e.g., ELISA).

Lastly, the dissection procedure could potentially be modified for accurate extraction of other brain regions, also for research questions not related to neurogenesis. For instance, CSD includes a brief semi-thawing step, during which compact myelin is visible as white areas distinct from the more translucent residual brain tissue. With a simple modification of the method, this feature would allow the precise dissection of only corpus callosum compact myelin tissue, which could be subjected to proteomic analysis of injury-related changes. A suggestion of a protocol modification that would allow the corpus callous dissection is to omit steps 1.5-1.9 of the protocol and proceed directly to preparing the coronal sections instead of opening the ventricles to make the SEZ and MEZ accessible. Then, place the sections on dry ice, briefly lift and semi-thaw the slices, and simply remove the corpus callosum with a scalpel. This preparation should now be ready for any analysis requiring an efficient dissection of native corpus callosum tissue.

In summary, this study presents a micro-dissection method that could be used for reliable ventricular neurogenic niche proteome analysis. The data underline the compatibility and utility of the CSD method together with MS-based proteomic analysis of the SEZ microenvironment. The combination of precision, efficiency, and minimal tissue perturbation render the CSD a valuable extension of existing methods.

\section{Disclosures}

The authors declare no competing interests

\section{Acknowledgments}

We wish to sincerely thank Mathias Mann for allowing us to perform large parts of the experiments in his laboratory, Fabian Coscia for help with LCM and proteome analysis, Tatiana Simon-Ebert for wholemount dissections, and Korbinian Mayr and Igor Paron for their technical help. We gratefully acknowledge funding from the German Research Council to MG (SFB870, TFR274), the EU (Eranet S-700982-5008-001), the ERC (aERC "NeuroCentro" to MG), the Swedish Society for Medical Research (SSMF, to JK) postdoctoral grant, and $\mathrm{KI}$ foundations and Funds (2020-01351, to JK).

\section{References}

1. Goodman, T., Hajihosseini, M. K. Hypothalamic tanycytes-masters and servants of metabolic, neuroendocrine, and neurogenic functions. Frontiers in Neuroscience. 9, 387 (2015). 
2. Chaker, Z. et al. Hypothalamic neurogenesis persists in the aging brain and is controlled by energy-sensing IGFI pathway. Neurobiology of Aging. 41, 64-72 (2016).

3. Kuhn, H. G., Toda, T., Gage, F. H. Adult hippocampal neurogenesis: a coming-of-age story. Journal of Neuroscience. 38 (49), 10401-10410 (2018).

4. Obernier, K., Alvarez-Buylla, A. Neural stem cells: origin, heterogeneity and regulation in the adult mammalian brain. Development. 146 (4), dev.156059 (2019).

5. Obernier, K. et al. Adult neurogenesis is sustained by symmetric self-renewal and differentiation. Cell Stem Cell. 22 (2), 221-234.e8 (2018).

6. Bordiuk, O. L., Smith, K., Morin, P. J., Semënov, M. V. Cell proliferation and neurogenesis in adult mouse brain. PloS One. 9 (11), e111453 (2014).

7. Doetsch, F., García-Verdugo, J. M., Alvarez-Buylla, A. Cellular composition and three-dimensional organization of the subventricular germinal zone in the adult mammalian brain. Journal of Neuroscience. 17 (13), 5046-5061 (1997).

8. Lim, D. A., Alvarez-Buylla, A. The adult ventricularsubventricular zone (V-SVZ) and olfactory bulb (OB) neurogenesis. Cold Spring Harbor Perspectives in Biology. 8 (5), a018820 (2016).

9. Sato, K. Effects of microglia on neurogenesis. Glia. 63 (8), 1394-1405 (2015).

10. Tavazoie, M. et al. A specialized vascular niche for adult neural stem cells. Cell Stem Cell. 3 (3), 279-288 (2008).

11. Sirko, S. et al. Chondroitin sulfates are required for fibroblast growth factor-2-dependent proliferation and maintenance in neural stem cells and for epidermal growth factor-dependent migration of their progeny. Stem Cells. 28 (4), 775-787 (2010).

12. Mercier, F. Fractones: extracellular matrix niche controlling stem cell fate and growth factor activity in the brain in health and disease. Cellular and Molecular Life Sciences: CMLS. 73 (24), 4661-4674 (2016).

13. Mercier, F., Kitasako, J. T., Hatton, G. I. Anatomy of the brain neurogenic zones revisited: fractones and the fibroblast/macrophage network. Journal of Comparative Neurology. 451 (2), 170-188 (2002).

14. Nascimento, M. A., Sorokin, L., Coelho-Sampaio, T. Fractone bulbs derive from ependymal cells and their laminin composition influence the stem cell niche in the subventricular zone. Journal of Neuroscience. 38 (16), 3880-3889 (2018).

15. Chen, G. et al. In vivo reprogramming for brain and spinal cord repair. eNeuro. 2 (5), ENEURO.0106-15.2015 (2015).

16. Zhang, Q., Chen, W., Tan, S., Lin, T. Stem cells for modeling and therapy of Parkinson's disease. Human Gene Therapy. 28 (1), 85-98 (2017).

17. Wei, C., Xiong, S., Cheng, L. Reprogramming of fibroblasts to neural stem cells by a chemical cocktail. Methods in Molecular Biology. 2117, 265-270 (2020).

18. Tian, Z., Zhao, Q., Biswas, S., Deng, W. Methods of reactivation and reprogramming of neural stem cells for neural repair. Methods. 133, 3-20 (2018).

19. Heinrich, C. et al. Sox2-mediated conversion of NG2 glia into induced neurons in the injured adult cerebral cortex. Stem Cell Reports. 3 (6), 1000-1014 (2014).

20. Masserdotti, G. et al. Transcriptional mechanisms of proneural factors and REST in regulating neuronal 
reprogramming of astrocytes. Cell Stem Cell. 17 (1), 74-88 (2015).

21. Seidenfaden, R., Desoeuvre, A., Bosio, A., Virard, I., Cremer, H. Glial conversion of SVZ-derived committed neuronal precursors after ectopic grafting into the adult brain. Molecular and Cellular Neurosciences. 32 (1-2), 187-198 (2006).

22. Lepko, T. et al. Choroid plexus-derived miR-204 regulates the number of quiescent neural stem cells in the adult brain. EMBO Journal. 38 (17), e100481 (2019).

23. Silva-Vargas, V., Maldonado-Soto, A. R., Mizrak, D., Codega, P., Doetsch, F. Age-dependent niche signals from the choroid plexus regulate adult neural stem cells. Cell Stem Cell. 19 (5), 643-652 (2016).

24. Angelidis, I. et al. An atlas of the aging lung mapped by single cell transcriptomics and deep tissue proteomics. Nature Communications. 10 (1), 963 (2019).

25. Kjell, J. et al. Defining the adult neural stem cell niche proteome identifies key regulators of adult neurogenesis. Cell Stem Cell. 26 (2), 277-293.e8 (2020).

26. Mirzadeh, Z., Doetsch, F., Sawamoto, K., Wichterle, H., Alvarez-Buylla, A. The subventricular zone en-face: wholemount staining and ependymal flow. Journal of Visualized Experiments: JoVE. (39), 1938 (2010).

27. Kulak, N. A., Geyer, P. E., Mann, M. Loss-less nano-fractionator for high sensitivity, high coverage proteomics. Molecular \& Cellular Proteomics: MCP. 16 (4), 694-705 (2017).

28. Tyanova, S., Temu, T., Cox, J. The MaxQuant computational platform for mass spectrometry-based shotgun proteomics. Nature Protocols. 11 (12), 2301-2319 (2016).
29. Cox, J., Mann, M. MaxQuant enables high peptide identification rates, individualized p.p.b.-range mass accuracies and proteome-wide protein quantification. Nature Biotechnology. 26 (12), 1367-1372 (2008).

30. Cox, J. et al. Accurate proteome-wide label-free quantification by delayed normalization and maximal peptide ratio extraction, termed MaxLFQ. Molecular \& Cellular Proteomics: MCP. 13 (9), 2513-2526 (2014).

31. Perez-Riverol, Y. et al. The PRIDE database and related tools and resources in 2019: improving support for quantification data. Nucleic Acids Research. 47 (D1), D442-D450 (2019).

32. Tomassy, G. S. et al. Distinct profiles of myelin distribution along single axons of pyramidal neurons in the neocortex. Science. 344 (6181), 319-324 (2014).

33. Sharma, K. et al. Cell type- and brain region-resolved mouse brain proteome. Nature Neuroscience. 18 (12), 1819-1831 (2015).

34. Datta, S. et al. Laser capture microdissection: Big data from small samples. Histology and Histopathology. 30 (11), 1255-1269 (2015).

35. Kerever, A. et al. Novel extracellular matrix structures in the neural stem cell niche capture the neurogenic factor fibroblast growth factor 2 from the extracellular milieu. Stem Cells. 25 (9), 2146-2157 (2007).

36. Cox, J., Mann, M. 1D and 2D annotation enrichment: a statistical method integrating quantitative proteomics with complementary high-throughput data. BMC Bioinformatics. 13 (Suppl 16), S12 (2012).

37. Daynac, M., Morizur, L., Chicheportiche, A., Mouthon, M. -A., Boussin, F. D. Age-related neurogenesis decline in the subventricular zone is associated with specific cell 
cycle regulation changes in activated neural stem cells.

Scientific Reports. 6, 21505 (2016).

38. Navarro Negredo, P., Yeo, R. W., Brunet, A. Aging and rejuvenation of neural stem cells and their niches. Cell Stem Cell. 27 (2), 202-223 (2020).

39. Smith, L. K., White, C. W., Villeda, S. A. The systemic environment: at the interface of aging and adult neurogenesis. Cell and Tissue Research. 371 (1), 105-113 (2018).

40. Neuberger, E. J., Swietek, B., Corrubia, L., Prasanna, A., Santhakumar, V. Enhanced dentate neurogenesis after brain injury undermines long-term neurogenic potential and promotes seizure susceptibility. Stem Cell Reports. 9 (3), 972-984 (2017).

41. Fisch, U., Brégère, C., Geier, F., Chicha, L., Guzman, R. Neonatal hypoxia-ischemia in rat elicits a regionspecific neurotrophic response in SVZ microglia. Journal of Neuroinflammation. 17 (1), 26 (2020).

42. Götz, M., Sirko, S., Beckers, J., Irmler, M. Reactive astrocytes as neural stem or progenitor cells: In vivo lineage, In vitro potential, and Genome-wide expression analysis. Glia. 63 (8), 1452-1468 (2015).

43. Kernie, S. G., Parent, J. M. Forebrain neurogenesis after focal Ischemic and traumatic brain injury. Neurobiology of Disease. 37 (2), 267-274 (2010).

44. Pous, L. et al. Fibrinogen induces neural stem cell differentiation into astrocytes in the subventricular zone via BMP signaling. Nature Communications. 11 (1), 630 (2020). 